\title{
Optimal Duration of Spectrum Lease: A Mathematical Approach
}

\author{
Gourav Saha*, Alhussein A. Abouzeid ${ }^{* \dagger}$, Marja Matinmikko-Blue ${ }^{\dagger}$ \\ *Department of Electrical, Computer and Systems Engineering, Rensselaer Polytechnic Institute, Troy, NY 12180, USA \\ $\dagger$ Centre for Wireless Communications, University of Oulu, Finland \\ Email: sahag@rpi.edu, abouzeid@ecse.rpi.edu,Marja.Matinmikko@oulu.fi
}

\begin{abstract}
It is widely accepted that the lease duration of spectrum bands sold through auctions plays a crucial role in affecting spectrum utilization. Shorter lease duration increases the frequency of spectrum auctions. Within the scenario studied in this paper, frequent spectrum auctions increases the chances of the spectrum bands being allocated to those operators who can utilize it the best. However, if the lease duration is too short it will not incentivize enough operators to join the market and hence lead to lower competition which in turn deteriorates spectrum utilization. In spite of the importance of lease duration in affecting spectrum utilization, there is no formal study dealing with optimizing the duration of a spectrum lease. This paper presents the first mathematical model to study the effect of lease duration on spectrum utilization under certain market scenario. In such a market scenario, revenue of an operator is the measure of its spectrum utilization. Based on our model, we formulate an optimization problem and develop algorithms to solve the optimization problem for two special cases. Using these algorithms, we numerically study how the optimal characteristics vary with different market parameters.
\end{abstract}

Index Terms-Spectrum License, Lease Duration, Spectrum Utilization, Operators' Revenue, Optimization

\section{INTRODUCTION}

With the rapid growth of wireless services and devices, the wireless data traffic is increasing. Cisco's forecast [1] shows that there will be a 7-fold increase in global data traffic from 2016 to 2021 . There is only a finite amount of wireless spectrum that can be used to support the growing wireless data traffic. There are various reports [2], [3] that show that many licensed spectrum bands are underutilized, leading to inefficient use of spectrum bands. It is widely accepted that the legacy policy of static spectrum allocations is a major cause of inefficient spectrum utilization [4]. In static spectrum allocation, spectrum bands are leased on a long-term basis which can lead to spectrum hoarding [5]. The exorbitant cost of such long-term spectrum leases forms a barrier for smaller companies to enter the spectrum market. Such reasons reduce market competition and hence may lead to inefficient spectrum utilization. In the past decade, there have been several research directions to combat the issue of spectrum under-utilization, most of which can be classified under the umbrella term spectrum sharing.

This material is based upon work supported by the National Science Foundation under grant numbers CNS-1422153 and CNS-1456887, as well as by a FiDiPro Fellow award from Business Finland (MOSSAF) and Academy of Finland 6Genesis Flagship (grant 318927).
Although spectrum sharing can vastly improve spectrum utilization, the implementation and administration of static spectrum allocation is much easier compared to spectrum sharing. For static spectrum allocation, it is widely accepted that the duration of spectrum lease is a critical factor which affects spectrum utilization. In the recently proposed 3-Tier Spectrum Sharing Framework [6], lease duration for "Priority Access Licenses (PAL) tier" was an important topic of debate. Many academic research works on spectrum sharing start by pointing out that long lease duration of static allocation leads to under-utilization of spectrum [7], [8]. In spite of the importance of lease duration, there is no formal study dealing with optimizing the same.

In this paper, we present a mathematical model to study the optimal duration of spectrum leases when channels are allocated for exclusive use. Based on our model, we formulate an optimization problem whose objective is to maximize spectrum utilization. In our model, the revenue of an operator is the measure of its spectrum utilization. This holds under certain market scenarios which we justify in Section II-A. The optimization problem has only one scalar decision variable, the lease duration. Shorter lease duration increases the frequency of spectrum auctions. Within the scenario studied in this paper, frequent spectrum auctions increase the chances of the spectrum bands being allocated to those operators who can utilize it the best. However, if the lease duration is too short it will not incentivize enough operators to join the market and hence lead to lesser competition which in turn deteriorates spectrum utilization. This argument suggests that the optimization problem should have non-trivial solution. We explicitly point out that the aim of this work is not to design optimal lease duration mechanisms, because doing that requires certain private information about an operator which may not be possible. Rather, we simply aim to mathematical model and analyze the effect of lease duration on spectrum utilization if private information was available.

There have been several active areas of research related to spectrum licences, such as pricing [9], auction design [10], flexible licensing [11], enforcement [12], etc. To the best of authors' knowledge, there is no mathematical study to understand the impact of lease duration of spectrum licenses on spectrum utilization. Perhaps, there is no similar mathematical study even in other synergistic areas such as electricity markets and cloud computing. However, there are few works in the 
spectrum sharing literature that consider the effect of certain "duration aspects" on the overall performance of the system. [13] considers a market of only two service providers with a common customer base. Time is divided into intervals. At the beginning of every interval, an auction is conducted which redistributes the available bandwidth based on the bids of individual service providers. The ratio of the customer demand reaching each service provider is governed by evolutionary game theory. The authors use simulations to conclude that shorter allocation interval corresponds to better spectrum utilization. In [14], the authors model various factors that a secondary service provider considers when buying spectrum resources from primary service providers. The authors design a utility function for the secondary service provider which suggests that longer contract duration is better. In [15], the primary user leases its bandwidth to secondary users for a fraction of time in exchange for cooperation (relaying). If the fraction of time is too small, it will not compensate for the overall cost of transmission (including relaying) and hence the secondary users may not agree to cooperate. For opportunistic spectrum use, optimal spectrum sensing time is an area which received wide attention from the spectrum community [16], [17]. There are few works in economic journals that consider the problem of optimizing contract duration for welfare analysis [18], [19]. The fundamental idea governing these works is a tradeoff between opportunity cost and transaction cost. The definitions of transaction cost and opportunity cost change with the market setting, like housing property market [20], priority service market [21], etc.

The paper outline and key contributions can be summarized as follows. In Section II-A we present a system model which can be used to study the effect of lease duration on spectrum utilization. Though simple, our model captures key aspects of spectrum leasing: 1) Improved spectrum utilization with a decrease in lease duration. 2) Improved spectrum utilization with more competition. 3) Why a smaller operator may prefer shorter lease duration. These aspects are discussed in detail in Section II-C. We are the first to propose such a system model to study optimal lease duration. This constitutes the first contribution of the paper.

We formulate the optimization problem in Section II-B. The optimization problem has one scalar decision variable, the lease duration. Yet, it is not trivial because is reminiscent of combinatorial optimization. To elaborate, the key idea behind the optimization problem is to use lease duration as a control knob that implicitly decides which wireless operators enter the market. This constitutes the second contribution of the paper.

We design algorithms to solve the optimization problem for two special cases in Section III. Since the optimization problem has a combinatorial nature, the number of candidate solutions may be exponential in the number of operators in the market. The design of the optimization algorithm relies on the result that for the two special cases studied in this paper, the number of candidate solutions is linear in the number of operators in the market. Designing the optimization algorithm is the third contribution of the paper. The final contribution

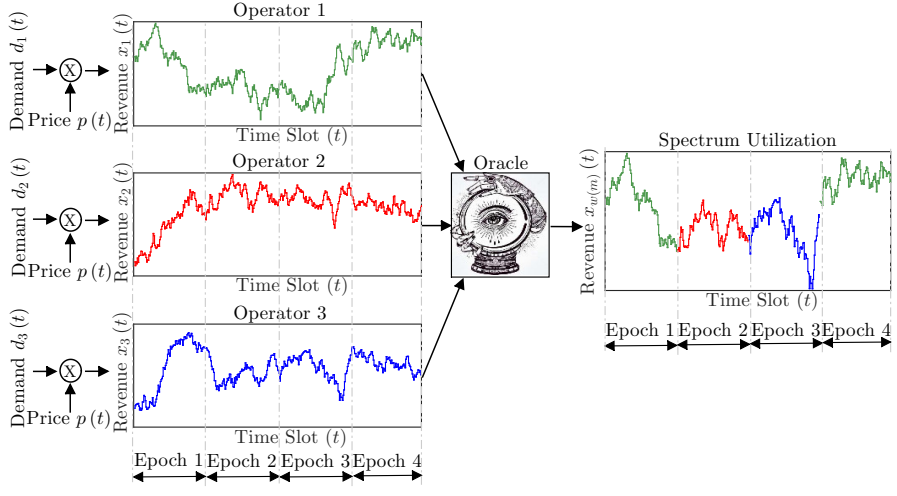

Fig. 1. Pictorial representation of certain critical elements of our system model. There are three operators, i.e. $n=3$. In the $t^{t h}$ time slot, all the operators charge $p(t)$ to serve a unit of customer demand. Hence, the revenue of the $k^{t h}$ operator is $x_{k}(t)=p(t) d_{k}(t)$. The channel is allocated to Operator 1 in the $1^{\text {st }}$ and the $4^{t h}$ epoch $(w(1)=w(4)=1)$, Operator 2 in the $2^{\text {nd }}$ epoch $(w(2)=2)$ and Operator 3 in the $3^{\text {rd }}$ epoch $(w(3)=3)$.

of the paper is the numerical results presented in Section IV. We use our optimization algorithm to study the optimal characteristics, i.e. optimal lease duration and optimal value of the objective function, as a function of market parameters.

\section{PRoblem Formulation}

We start by presenting our system model in Section II-A. To develop a tractable system model, we make various assumptions which are then justified with appropriate reasons. By making these assumptions, we abstract away from several complexities which allows us to focus solely on the impact of lease duration on spectrum utilization. In Section II-B, we use the system model to formulate an optimization problem to maximize spectrum utilization. In doing so, we introduce the objective function and the revenue function, which capture spectrum utilization and the revenue of an operator, respectively. Finally, we study the properties of the objective and the revenue function in Section II-C which reveal various important aspects that can be captured by our system model.

\section{A. System Model}

We consider a time slotted model where a time slot is denoted by $t \in\{1,2, \ldots\}$. Let $T$ denote the lease duration. The word epoch denotes an entire lease duration. Hence, the time slots corresponding to the $m^{\text {th }}$ epoch is $t \in[(m-1) T+1, m T]$ where $m \in\{1,2, \ldots\}$. There are $n$ operators indexed $k=1,2, \ldots, n$. There is only one channel which is allocated to one of these operators, in the beginning of every epoch, for exclusive use.

In our model, the revenue generated by an operator is a measure of its spectrum utilization. This can be justified by first relating spectrum utilization of an operator with its customer demand and then relating customer demand with the operator's revenue. The justification is as follows: First, the amount of customer demand served by an operator is a measure of its spectrum utilization. Even though channel throughput is the most commonly used measure of spectrum 
TABLE I

A TABLE OF FREQUENTLY USED NOTATIONS.

\begin{tabular}{|c|c|}
\hline Notation & Description \\
\hline$t$ & $t^{t h}$ time slot. \\
\hline $\bar{T}$ & Lease duration. \\
\hline$x_{k}(t)$ & Revenue of the $k^{t h}$ operator in $t^{t h}$ time slot. \\
\hline$\mu_{k}$ & $\begin{array}{l}\text { Mean of the revenue process of the } k^{t h} \text { operator, } \\
E\left[x_{k}(t)\right]=\mu_{k} ; \forall t\end{array}$ \\
\hline$\sigma_{k}$ & $\begin{array}{l}\text { Standard deviation of the revenue process of the } k^{t h} \text { operator, } \\
\sqrt{E\left[\left(x_{k}(t)-\mu_{k}\right)^{2}\right]}=\sigma_{k} ; \forall t\end{array}$ \\
\hline$a_{k}$ & $\begin{array}{l}\text { Autocorrelation coefficient of the revenue process of the } k^{t h} \\
\text { operator. We have } a_{k} \in(0,1) \text {. }\end{array}$ \\
\hline$R_{k}^{m}$ & MERR of the $k^{t h}$ operator. \\
\hline $\bar{T}_{k}$ & $\begin{array}{l}\text { Minimum lease duration to satisy the MERR of the } k^{t h} \\
\text { operator if it was alone in the market. We have } \bar{T}_{k}=\frac{R_{k}^{m}}{\mu_{k}} \text {. }\end{array}$ \\
\hline $\mathcal{S}$ & Set of interested operators. \\
\hline$s$ & Number of interested operators. We have $s=|\mathcal{S}|$. \\
\hline $\mathcal{R}_{k}(T)$ & $\begin{array}{l}\text { Revenue function of the } k^{t h} \text { operator as a function of lease } \\
\text { duration } T \text {. }\end{array}$ \\
\hline $\mathcal{R}_{k}(\mathcal{S}, T)$ & $\begin{array}{l}\text { Revenue function of the } k^{t h} \text { operator as a function of set of } \\
\text { interested operators } \mathcal{S} \text { and lease duration } T \text {. }\end{array}$ \\
\hline $\mathcal{R}(s, T)$ & $\begin{array}{l}\text { Revenue function of an operator as a function of number of } \\
\text { interested operators } s \text { and lease duration } T \text {. It only applies } \\
\text { for a market that is homogeneous in in } \mu_{k}, \sigma_{k} \text { and } a_{k} \text {. }\end{array}$ \\
\hline$U(T)$ & Objective function as a function of lease duration $T$. \\
\hline$U(\mathcal{S}, T)$ & $\begin{array}{l}\text { Objective function as a function of set of interested operators } \\
\mathcal{S} \text { and lease duration } T \text {. }\end{array}$ \\
\hline$U(s, T)$ & $\begin{array}{l}\text { Objective function as a function of number of interested } \\
\text { operators } s \text { and lease duration } T \text {. It only applies for a market } \\
\text { that is homogeneous in } \mu_{k}, \sigma_{k} \text { and } a_{k} \text {. }\end{array}$ \\
\hline
\end{tabular}

utilization, there are studies like [22], [23] that used other metrics as a measure of spectrum utilization. Second, according to Bertrand and Cournot competition models [24], [25], with two or more operators, the market reaches perfect competition and all operators sell at the same price. Similar results are shown to hold in [26], [27]. Since all operators sell at the same price, an operator with a higher revenue serves more customer demand and hence utilizes its channel better (see Figure 1). The $k^{t h}$ operator's revenue at time slot $t$ is $x_{k}(t)$ if the operator has the channel and 0 otherwise. We assume that $x_{k}(t)$ is a first order Gaussian autoregressive process [28], [29],

$$
x_{k}(t+1)=a_{k} x_{k}(t)+\varepsilon_{k}(t) ; \forall t \geq 1, k
$$

where $a_{k} \in(0,1)$ is the autocorrelation coefficient and $\varepsilon_{k}(t)$ is an iid Gaussian random process with mean $\mu_{k}^{\varepsilon}$ and standard deviation $\sigma_{k}^{\varepsilon}$, i.e. $\varepsilon_{k}(t) \sim \mathcal{N}\left(\mu_{k}^{\varepsilon}, \sigma_{k}^{\varepsilon}\right), \forall t$. It can be shown that $x_{k}(t)$ is a stationary Gaussian random process [30] with mean $\mu_{k}$ and standard deviation $\sigma_{k}$, i.e. $x_{k}(t) \sim \mathcal{N}\left(\mu_{k}, \sigma_{k}\right), \forall t$ where

$$
\mu_{k}=\frac{\mu_{k}^{\varepsilon}}{1-a_{k}} ; \sigma_{k}=\frac{\sigma_{k}^{\varepsilon}}{\sqrt{1-a_{k}^{2}}}
$$

Concerning channel allocation, our model considers that there is an oracle which can see the future revenues of all the $n$ operators (see Figure 1). In the $m^{t h}$ epoch, the oracle allocates/leases the channel to the operator with the highest total revenue in the $m^{t h}$ epoch; $\sum_{t=(m-1) T+1}^{m T} x_{k}(t)$. The cost of leasing the channel is chosen to be a constant.
This allocation strategy can be justified as follows. First, this strategy is insightful because it yields an upper bound on spectrum utilization as we are assuming full knowledge of future revenues. Such bounds may be very close to the true performance [31], [32]. Second, it decouples our model from any specific auction mechanism. For the same reason, the cost of leasing a channel is chosen to be a constant rather than being dependent on operators' bids. This is consistent with prior works, e.g., [33].

To enter the market, the $k^{\text {th }}$ operator has to invest in infrastructure development. In order to get a return on infrastructure development cost and the cost of leasing a channel, the $k^{\text {th }}$ operator wants to make a minimum expected revenue $R_{k}^{m}$ in an epoch. The $k^{t h}$ operator will not enter the market if the lease duration does not satisfy its minimum expected revenue requirement (MERR). As it will become clear in Section II-B and II-C, the revenue of an operator depends on the value of $\mu_{k}, \sigma_{k}, a_{k}$ and $R_{k}^{m}$ of all the operators in the market. Our model assumes that an operator has full information of $\mu_{k}$, $\sigma_{k}, a_{k}$ and $R_{k}^{m}$ of all the operators using which it decides whether to enter the market or not. A similar assumption is made in prior works, such as [34] and references therein, for mathematical tractability.

Table I lists the notations used throughout this paper. Other notations used in this paper are standard.

\section{B. The Optimization Problem}

As mentioned in Section II-A, an operator will only enter the market if its MERR is met. Let $\mathcal{S}(T) \subseteq\{1,2, \ldots, n\}$ be the subset operators who enters the market if lease duration is $T$. The oracle allocates the channel among the operators in set $\mathcal{S}(T)$ according to the channel allocation strategy,

$$
w(m)=\arg \max _{k \in \mathcal{S}(T)} \sum_{t=1}^{T} x_{k}((m-1) T+t)
$$

where $w(m)$ denotes the index of the operator who is allocated the channel in the $m^{\text {th }}$ epoch. Figure 1 shows an example of such a channel allocation strategy.

Let $\mathcal{R}_{k}(T)$ denote the expected revenue of the $k^{\text {th }}$ operator in an epoch if the lease duration is $T . \mathcal{R}_{k}(T)$ is called the revenue function of the $k^{t h}$ operator. Since $x_{k}(t)$ is a stationary process, the revenue function of the $k^{\text {th }}$ operator is equal in every epoch, i.e. $\mathcal{R}_{k}(\cdot)$ does not depend on $m$. Therefore, we can just consider the $1^{\text {st }}$ epoch to derive an expression for $\mathcal{R}_{k}(T)$. We have,

$$
\begin{aligned}
\mathcal{R}_{k}(T) & =E\left[\sum_{t=1}^{T} x_{k}(t) \mid I_{k}=1\right] P\left[I_{k}=1\right]+0 \cdot P\left[I_{k}=0\right] \\
& =E\left[\sum_{t=1}^{T} x_{k}(t) \mid I_{k}=1\right] P\left[I_{k}=1\right]
\end{aligned}
$$

where $I_{k}$ is an indicator variable which is 1 if the $k^{\text {th }}$ operator is allocated the channel and 0 otherwise. In (4), $E\left[\sum_{t=1}^{T} x_{k}(t) \mid I_{k}=1\right]$ is the net expected revenue of the $k^{t h}$ operator in an epoch if the channel is allocated to the $k^{t h}$ 
operator. The net expected revenue of the $k^{t h}$ operator in an epoch is 0 if the channel is not allocated to the $k^{\text {th }}$ operator.

Proposition 1. If channel allocation is governed by (3) and,

$$
\begin{aligned}
\mu_{i}^{S}(T) & =\mu_{i} T \\
\sigma_{i}^{S}(T) & =\frac{\sqrt{T-a_{i}\left(2-2 a_{i}^{T}+a_{i} T\right)}}{\left(1-a_{i}\right)} \sigma_{i} \\
f_{i}(\theta, T) & =\frac{1}{\sqrt{2 \pi} \sigma_{i}^{S}(T)} \exp \left(-\frac{\left(\theta-\mu_{i}^{S}(T)\right)^{2}}{2 \sigma_{i}^{S}(T)^{2}}\right) \\
F_{i}(\theta, T) & =\frac{1}{2}\left(1+\operatorname{erf}\left(\frac{\theta-\mu_{i}^{S}(T)}{\sqrt{2} \sigma_{i}^{S}(T)}\right)\right)
\end{aligned}
$$

where $\operatorname{erf}(\cdot)$ is the error function, then the revenue function of the $k^{\text {th }}$ operator, where $k \in \mathcal{S}(T)$, is

$$
\mathcal{R}_{k}(T)=\int_{-\infty}^{\infty} \theta\left(\prod_{j \neq k, j \in \mathcal{S}(T)} F_{j}(\theta, T)\right) f_{k}(\theta, T) d \theta
$$

Proof: Please refer to Appendix A for the proof.

According to our system model, the $k^{\text {th }}$ operator will enter the market only if $\mathcal{R}_{k}(T) \geq R_{k}^{m}$. Hence, a necessary condition (not sufficient) $\mathcal{S}(T)$ must satisfy is,

$$
\mathcal{S}(T)=\left\{k: \mathcal{R}_{k}(T) \geq R_{k}^{m}\right\}
$$

A few remarks are in order. First, note that $\mathcal{R}_{k}(T)$ is dependent on $\mathcal{S}(T)$ (see (9)) which in turn is dependent on $\mathcal{R}_{k}(T)$ (refer to (10)). Second, the term $\prod_{j \neq k, j \in \mathcal{S}(T)} F_{j}(\theta, T)$ of (9) clearly shows that the revenue of the $k^{t h}$ operator depends on other operators in the market. In particular, $F_{j}(\theta, T)$ and $\mathcal{S}(T)$ depend on $\mu_{j}, \sigma_{j}, a_{j}$ and $R_{j}^{m}$ (see (8), (5), (6) and (10)). Therefore, an operator needs the knowledge of $\mu_{j}, \sigma_{j}$ , $a_{j}$ and $R_{j}^{m}$ of all the operators in the market in order to calculate its expected revenue in an epoch and hence decide whether to enter the market or not.

Our objective is to maximize the net expected revenue $V$ (equivalent to net expected spectrum utilization) in optimization horizon $\mathcal{T} \gg T$. Assume that $\mathcal{T}$ is a multiple of $T$, i.e $\mathcal{T}=M T$ where $M$ is an integer. Mathematically,

$$
V=\sum_{m=1}^{M} E\left[\sum_{t=1}^{T} x_{w(m)}((m-1) T+t)\right]
$$

In (11), $E\left[\sum_{t=1}^{T} x_{w(m)}((m-1) T+t)\right]$ denotes the expected revenue in the $m^{\text {th }}$ epoch where the expectation is over the random process $x_{k}(t)$ and the random variable $w(m)$. Given that $x_{k}(t)$ is stationary, the term $E\left[\sum_{t=1}^{T} x_{w(m)}((m-1) T+t)\right]$ is independent of $m$. In other words, the net expected revenue is equal in all epochs. Hence, (11) can be simplified to

$$
V=M E\left[\sum_{t=1}^{T} x_{w(1)}(t)\right]=\mathcal{T} \frac{E\left[\sum_{t=1}^{T} x_{w(1)}(t)\right]}{T}
$$

Equation 12 shows that maximizing $V$ is equivalent to maximizing $\frac{E\left[\sum_{t=1}^{T} x_{w(1)}(t)\right]}{T}$. This holds even if $\mathcal{T}$ is not a multiple of $T$ provided $\mathcal{T} \gg T$. The objective function of our optimization problem is

$$
\begin{aligned}
U(T) & =\frac{E\left[\sum_{t=1}^{T} x_{w(1)}(t)\right]}{T} \\
& =\frac{\sum_{k \in \mathcal{S}(T)} E\left[\sum_{t=1}^{T} x_{k}(t) \mid I_{k}=1\right] P\left[I_{k}=1\right]}{T} \\
& =\frac{1}{T} \sum_{k \in \mathcal{S}(T)} \mathcal{R}_{k}(T)
\end{aligned}
$$

Equation 14 is obtained using the law of iterated expectation over all possible $w(1)$ in (13). Equation 15 is obtained by observing that $E\left[\sum_{t=1}^{T} x_{k}(t) \mid I_{k}=1\right] P\left[I_{k}=1\right]$ is equal to $\mathcal{R}_{k}(T)$ (see (4)). Finally, our optimization problem is

$$
\text { OP1 }\left\{\max _{T} \quad U(T)=\frac{1}{T} \sum_{k \in \mathcal{S}(T)} \mathcal{R}_{k}(T)\right.
$$

In OP1, we can implicitly control $\mathcal{S}(T)$ by choosing a suitable $T$. A significant part of $\mathbf{O P} 1$ is to find the optimal combination of interested operators $\mathcal{S}(T)$ that maximizes the objective function $U(T)$. The number of combinations of $\mathcal{S}(T)$ is exponential in $n$. Therefore, OP1 is reminiscent of combinatorial optimization which makes it non-trivial even though it is a scalar optimization problem in $T$.

\section{Properties of the Objective and the Revenue Function}

We start this section by introduction two new functions,

$$
\begin{aligned}
\mathcal{R}_{k}(\mathcal{S}, T) & =\int_{-\infty}^{\infty} \theta\left(\prod_{j \neq k, j \in \mathcal{S}} F_{j}(\theta, T)\right) f_{k}(\theta, T) d \theta \\
U(\mathcal{S}, T) & =\frac{1}{T} \sum_{k \in \mathcal{S}} \mathcal{R}_{k}(\mathcal{S}, T)
\end{aligned}
$$

$\mathcal{R}_{k}(\mathcal{S}, T)$ and $U(\mathcal{S}, T)$ are the expected revenue of the $k^{t h}$ operator in an epoch and the value of the objective function, respectively, if the lease duration is $T$ and the set of interested operators is $\mathcal{S}$. Although $\mathcal{S}$ is not an independent variable, these functions are helpful in proving few properties of the revenue and objective function. Also, they are used in Section III to design optimization algorithms for OP1. If the market is homogeneous ${ }^{1}$ in $\mu_{k}, \sigma_{k}$ and $a_{k}$, i.e. $\mu_{k}=\mu, \sigma_{k}=\sigma$ and $a_{k}=a$ for all $k$ 's, then (17) and (18) simplifies to,

$$
\begin{aligned}
\mathcal{R}(s, T) & =\int_{-\infty}^{\infty} \theta F(\theta, T)^{s-1} f(\theta, T) d \theta \\
U(s, T) & =\frac{s}{T} \mathcal{R}(s, T)
\end{aligned}
$$

\footnotetext{
${ }^{1}$ The market may be heterogeneous in $R_{k}^{m}$.
} 


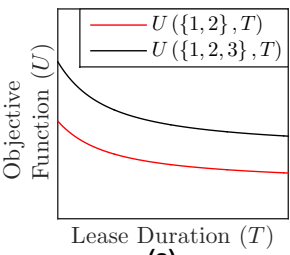

(a)

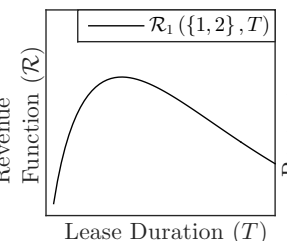

(b)

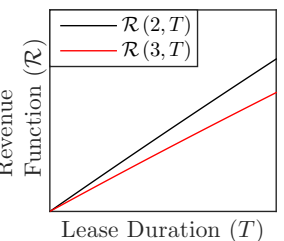

(c)
Fig. 2. Typical trends of the Objective and the Revenue Function. In (a) and (b), $\{1,2\}$ and $\{1,2,3\}$ are the values of $\mathcal{S}$, the set of interested operators, where 1, 2 and 3 are indices of the operators. In (c), 1, 2 and 3 are the values of $s$, the number of interested operators.

where $s=|\mathcal{S}|$ is the number of interested operators. Unlike (17), the functions $\mathcal{R}(s, T), F(\theta, T)$ and $f(\theta, T)$ of (19) does not have any subscript because the market is homogeneous. For the same reason, the revenue and the objective function depend only on the number of operators $s$ in the market. In the following, we discuss few properties of these functions.

Property 1. $U(\mathcal{S}, T)$ is monotonic non-increasing in $T$.

Property 2. $U(\mathcal{S}, T)$ is monotonic non-decreasing in $\mathcal{S}$, i.e. $U(\mathcal{S}, T) \leq U(\mathcal{S} \cup\{a\}, T)$ where $a \notin \mathcal{S}$.

Property 3. $\mathcal{R}(s, T)$ is monotonic non-decreasing in $T$.

Property 4. $\mathcal{R}(s, T)$ is monotonic non-increasing in $s$ if $s \geq 2$.

Proofs of these properties are deferred to [35] due to page limitation. Figure 2 is a pictorial representation of these properties. Figure 2.a. depicts Property 1 and 2 . The objective function $U(\mathcal{S}, T)$ decreases with increase in $T$ (both red and blue curves decrease). This is because with frequent spectrum allocation (or shorter lease duration), we are selecting the best operator more often. Also, the objective function increases as the set of interested operators $\mathcal{S}$ grows (black curve is above the red curve). Qualitatively, as $\mathcal{S}$ grows, competition increases, which in turn leads to better spectrum utilization (or higher value of objective function). Properties 1 and 2 extend to $U(s, T)$ because it is a special case of $U(\mathcal{S}, T)$.

For a heterogeneous market, the revenue function $\mathcal{R}(\mathcal{S}, T)$ does not have any monotonic properties like the objective function. A typical shape of the revenue function of Operator1 when there are two interested operators, Operator-1 and Operator-2, is shown in Figure 2.b. Such a case arises when Operator- 1 has a lower mean than Operator-2, i.e. $\mu_{1}<\mu_{2}$. In such a case, the revenue function of Operator-1 will start decreasing after a certain lease duration. This is because as $T$ increases, $\frac{\sum_{t=1}^{T} x_{k}((m-1) T+t)}{T} \rightarrow \mu_{k}$ due to the law of large numbers. Therefore, based on (3), the operator with the higher mean (Operator-2) will be allocated the channel most of the time. This shows that not all the operators prefer long lease duration. In a market that is homogeneous in $\mu_{k}$, $\sigma_{k}$ and $a_{k}$, the revenue function $\mathcal{R}(s, T)$ have monotonic properties. Figure 2.c. depicts Property 3 and 4. The revenue function increases with increase in $T$ (both red and blue curve increases) because the operators can make more revenue in an epoch as lease duration increases. Also, the revenue function decreases with increase in number of interested operators $s$ (black curve is above the red curve). This is because the probability of an operator being allocated a channel decreases ${ }^{2}$ with increase in $s$.

\section{Optimal Solution of the Optimization Problem}

In our model, an operator is fully characterized by four parameters: mean revenue $\mu_{k}$, standard deviation of revenue $\sigma_{k}$, autocorrelation constant $a_{k}$ and the MERR $R_{k}^{m}$. In this section, we concentrate on solving OP1 for two cases. First, in a homogeneous market where all the operators have the same parameters, i.e. $\mu_{k}=\mu, \sigma_{k}=\sigma, a_{k}=a$ and $R_{k}^{m}=R^{m}$ for all $k$ 's. Second, a special case of heterogeneous market where $\mu_{k}=\mu, \sigma_{k}=\sigma$ and $a_{k}=a$ for all $k$ 's. However, the MERR $R_{k}^{m}$ can vary with operator.

\section{A. Homogeneous Market}

In a homogeneous market, all the operators have the same revenue function (see (19)) and the same minimum expected revenue. Hence, for a given lease duration $T$, either all the $n$ operators are interested; i.e. $\mathcal{S}(T)=\{1,2, \ldots, n\}$, or none of the operators are interested, i.e. $\mathcal{S}(T)=\emptyset$. If none of the operators are interested, then the objective function $U(T)=0$. Therefore, we have to satisfy all the operators in order to maximize the objective function. With a little abuse of notation, let $\mathcal{R}(T)=\mathcal{R}(n, T)$ and $U(T)=U(n, T)$. In a homogeneous market, OP1 simplifies to

$$
\text { OP2 }\left\{\begin{array}{l}
\max _{T} U(T)=\frac{n}{T} \mathcal{R}(T) \\
\text { subject to: } \\
\mathcal{R}(T) \geq R^{m}
\end{array}\right.
$$

Since $U(T)$ is monotonic non-increasing in $T$ (Property 1), the optimal lease duration $T^{*}$ is the least $T$ satisfying the constraint $\mathcal{R}(T) \geq R^{m}$. Since $\mathcal{R}(T)$ is monotonic nondecreasing in $T$ (Property 3 ), the optimal lease duration $T^{*}$ must satisfy

$$
\mathcal{R}\left(T^{*}\right)=R^{m}
$$

Given that $\mathcal{R}(T)$ is monotonic in nature, (21) can be solved using binary-search or newton-raphson method to find the optimal lease duration $T^{*}$. Finally the optimal value of the objective function is

$$
U^{*}=U\left(T^{*}\right)=\frac{n}{T^{*}} \mathcal{R}\left(T^{*}\right)=\frac{n}{T^{*}} R^{m}
$$

\section{B. Heterogeneous Market: Special Case}

As the market is homogeneous in $\mu_{k}, \sigma_{k}$ and $a_{k}$, the revenue function of all the operators are equal and is given by (19). However, the MERR $R_{k}^{m}$ can vary with operator. In such a market, if $k^{\text {th }}$ operator is interested in joining the market, then so are other operators whose MERR is less than $R_{k}^{m}$. This is a critical observation which can be formalized as follows. Without any loss of generality, let $R_{j}^{m} \geq R_{i}^{m} ; \forall j>i$. Let $[s]=\{1, \ldots, s\}$ be the set of first $s$ operators.

\footnotetext{
${ }^{2}$ In a market that is homogeneous in $\mu_{k}, \sigma_{k}$ and $a_{k}$, the probability of an operator being allocated a channel is simply $\frac{1}{s}$.
} 


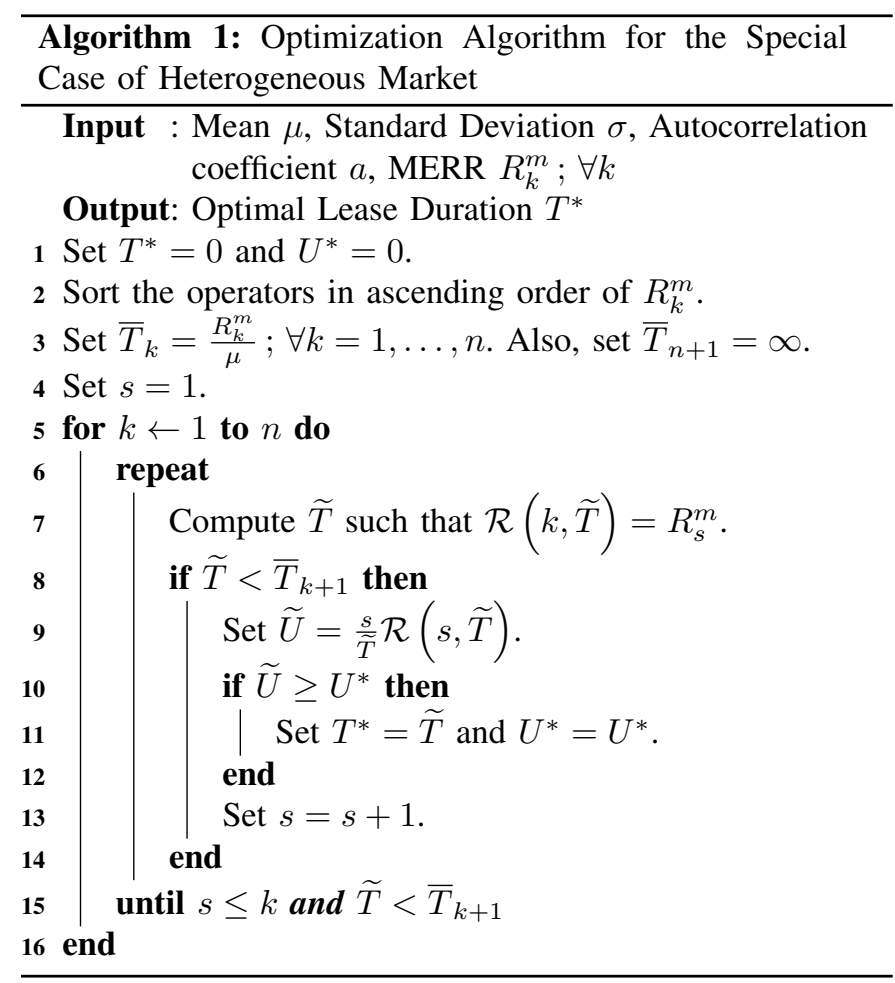

Proposition 2. If the market is homegenous in $\mu_{k}, \sigma_{k}$ and $a_{k}$, then $\mathcal{S}(T) \in \Omega$ where,

$$
\Omega=\{[s]: s=1, \ldots, n\}
$$

Proposition 2 is important because it reduces the number of combinations of $\mathcal{S}(T)$ from exponential to linear in $n$. Based on Proposition 2, we designed Algorithm 1 to find the optimal lease duration $T^{*}$ and optimal value of objective function $U^{*}$ for the special case of heterogeneous market. In rest of this section, we explain the working of Algorithm 1.

If $[s]$ is the set of interested operators, then according to (20), the value of the objective functions is $\widetilde{U}=\frac{s}{\widetilde{T}} \mathcal{R}(s, \widetilde{T})$ where $\widetilde{T}$ is the lease duration such that $\mathcal{S}(\widetilde{T})=[s] . \widetilde{U}$ is calculated in line 9 of Algorithm 1. For $[s]$ to be the set of interested operators, it is enough to ensure that the MERR of the $s^{t h}$ operator is satisfied. If $[s]$ is the set of interested operators, then the revenue function of an operator is given by (19). Therefore, to satisfy MERR of the $s^{\text {th }}$ operator, $\widetilde{T}$ must satisfy $\mathcal{R}(s, \widetilde{T}) \geq R_{s}^{m}$. Recall, $\widetilde{U}$ decreases with increase in $\widetilde{T}$ (Property 1) and $\mathcal{R}(s, \widetilde{T})$ increases with increase in $\widetilde{T}$ (Property 3 ). Therefore, to maximize $\widetilde{U}$, we can set $\widetilde{T}$ such that $\mathcal{R}(s, \widetilde{T})=R_{s}^{m}$. To this end, we have maximized $\widetilde{U}$ for a given $[s]$. But we don't know for which $[s]$ the value of $\widetilde{U}$ will be maximized. This is because as $s$ increases:

Statement 1: $\widetilde{U}$ increases (Property 2).

Statement 2: $\widetilde{T}$ increases which in turn decreases $\widetilde{U}$ (Property 1$)$. Increase in $\widetilde{T}$ with increase in $s$ can be explained as follows. As $s$ increases, $\mathcal{R}(s, \widetilde{T})$ decreases (Property 4). Therefore, to satisfy $\mathcal{R}(s, \widetilde{T})=R_{s}^{m}$, we need a larger $\widetilde{T}$.

Statements 1 and 2 show that an increase in $s$ has opposing affects on $\widetilde{U}$. Therefore, we calculate the maximum $\widetilde{U}$ for each $[s]$ and then choose the maximum among them. This is done in lines 10 and 11 of Algorithm 1.

Setting $\widetilde{T}$ such that $\mathcal{R}(s, \widetilde{T})=R_{s}^{m}$ will always not ensure that $[s]$ is the set of interested operators. Let $\bar{T}_{k}=\frac{R_{k}^{m}}{\mu}$ as calculated in line 3 of Algorithm 1. $\bar{T}_{k}$ is the minimum lease duration to satisfy the MERR of the $k^{t h}$ operator if it was alone in the market. If $\widetilde{T}$ satisfying $\mathcal{R}(s, \widetilde{T})=R_{s}^{m}$ is such that $\widetilde{T} \geq$ $\bar{T}_{s+1}$, then the $(s+1)^{t h}$ operator would also be interested in entering the market. If the $(s+1)^{t h}$ operator enters the market, the revenue function of an operator is $\mathcal{R}(s+1, \widetilde{T})$. Now using Property 4,

$$
\mathcal{R}(s+1, \widetilde{T}) \leq \mathcal{R}(s, \widetilde{T})=R_{s}^{m} \leq R_{s+1}^{m}
$$

Inequality 24 shows that if $\mathcal{R}(s, \widetilde{T})=R_{s}^{m}$ and $\widetilde{T} \geq \bar{T}_{s+1}$, then MERR of neither the $s^{\text {th }}$ operator nor the $(s+1)^{t h}$ operator will be satisfied. Hence, $[s]$ will not be the set of interested operators. Instead for $[s]$ to be the set of interested operators, $\widetilde{T}$ should be set such that MERR of $s^{\text {th }}$ operator is satisfied even if $(s+1)^{t h}$ operator enters the market. This can be done by setting $\widetilde{T}$ such that $\mathcal{R}(s+1, \widetilde{T})=R_{s}^{m}$. If such a $\widetilde{T} \geq \bar{T}_{s+2}$, then we have to loop until we find a $\widetilde{T}$ such that $\mathcal{R}(k, \widetilde{T})=R_{s}^{m}$ and $\widetilde{T}<\bar{T}_{k+1}$. This is implemented using the for and repeat-until loop of Algorithm 1. We guarantee the existence of such a $\widetilde{T}$ by setting a dummy variable $\bar{T}_{n+1}=\infty$ (refer line 3 of Algorithm 1). Please note that the equation $\mathcal{R}(k, \widetilde{T})=R_{s}^{m}$ in line 7 of Algorithm 1 can be solved using binary-search or newton-raphson method because $\mathcal{R}(k, \widetilde{T})$ is monotonic increasing in $\widetilde{T}$ (Property 3 ). Finally, the output of Algorithm 1 is the optimal lease duration $T^{*}$.

\section{Numerical Results}

In this section we use the optimization techniques from Section III to numerically study the effect of market parameters on optimal lease duration $T^{*}$ and optimal value of objective function $U^{*}$. Market parameters are the mean $\mu$, standard deviation $\sigma$, time constant ${ }^{3} \tau$ (where autocorrelation coefficient $a=\exp \left(-\frac{1}{\tau}\right)$ ), number of operators $n$ and MERR $R_{k}^{m}$. Default numerical parameters are $\mu=1, \sigma=1$, $\tau=500$ and $n=10$. These parameters are used unless otherwise stated. We divide the study of homogeneous market and special case of heterogeneous market into two subsections.

\section{A. Homogeneous Market}

In a homogeneous market, $R_{k}^{m}=R^{m} ; \forall k$. We use $R^{m}=$ 100 unless otherwise stated.

\footnotetext{
${ }^{3}$ Higher time constant implies higher autocorrelation.
} 

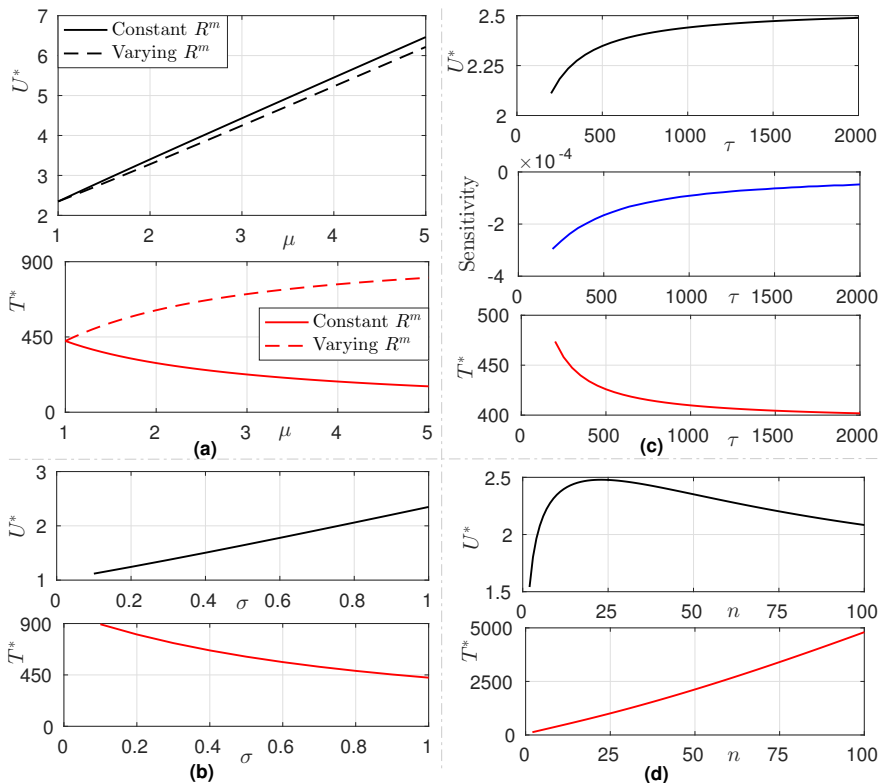

Fig. 3. Plots showing the variation of optimal characteristics $T^{*}$ and $U^{*}$ in a homogeneous market as a function of: (a) Mean $\mu$ (b) Standard deviation $\sigma$ (c) Time constant $\tau$ and (d) Number of operators $n$.

Effect of mean $\mu$ with constant MERR $R^{m}$ : As $\mu$ increases, an operator will earn more revenue in a given time slot. Therefore, it will take less time to generate the MERR $R^{m}$. Hence, optimal lease duration $T^{*}$ decreases as shown in Figure 3.a. (red solid line). Since $U^{*}$ is inversely proportional to $T^{*}$ according to (22), $U^{*}$ increases with increase in $\mu$. This is also shown in Figure 3.a. (black solid line).

Effect of mean $\mu$ with varying MERR $R^{m}$ : In this numerical study, $R^{m}=\alpha \mu$ where $\alpha>0$ (chosen as 100) is a constant. This numerical study answers the following question: "If the operators have higher $\mu$ but also have higher $R^{m}$, then is it better or worse for the value of $U^{*}$ ?". This is not trivial to answer because an increase in $\mu$ and $R^{m}$ have opposing affects on $U^{*}$. As shown in Figure 3.a. (red and black dashed lines), both $T^{*}$ and $U^{*}$ increases with increase in $\mu$. Please note that the relation between $T^{*}$ and $U^{*}$ can't be explained using (22) because $R^{m}$ is not a constant. To conclude, if the operators have higher $\mu$, they will improve $U^{*}$ even though their MERR is higher.

Effect of standard deviation $\sigma$ : As shown in Figure 3.b., $U^{*}$ increases with increase in $\sigma$. This can be explained as follows: If an operator is allocated the channel, then there is a high probability that the operator's revenue is more than the mean. This probability increases with increase in $\sigma$ because the operator's revenue fluctuates more around the mean. Hence $U^{*}$ increases with increase in $\sigma$. As $U^{*}$ increases, $T^{*}$ decreases according to (22).

Effect of time constant $\tau$ : Autocorrelation defines the selfsimilarity of a random process. As autocorrelation increases, an operator with higher revenue at current time slot will have higher revenue at a later time slot. This has two consequences. First, the operator who is allocated the channel will take less time to generate the MERR $R^{m}$. Hence, with increase in $\tau$,

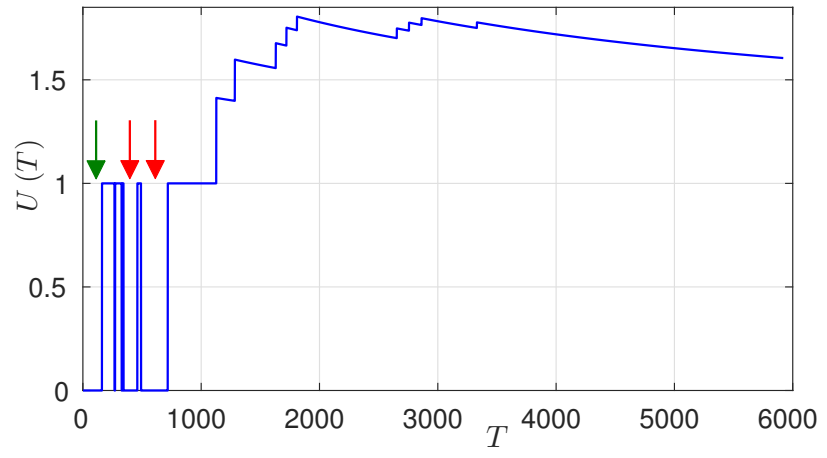

Fig. 4. A typical shape of objective function $U(T)$ for a particular instance of $R_{k}^{m}$. $R_{k}^{m}$ 's are choosen independently at random from the uniform distribution $\mathcal{U}(100,600)$.

optimal lease duration $T^{*}$ decreases which leads to increase in $U^{*}$ according to (22). This is shown in Figure 3.c. Second, the objective function becomes less sensitive to lease duration, i.e. it will not decrease increase significantly if the lease duration is higher. This is also shown in Figure 3.c. where sensitivity, defined as $\left.\frac{\partial U}{\partial T}\right|_{T=T^{*}}$, becomes less negative as $\tau$ increases.

Effect of number of operators $n$ : As the number of operators increases, the probability that a given operator is allocated the channel decreases. Therefore, to satisfy the MERR, the operators have to generate more revenue in an epoch when they are allocated the channel. Hence, optimal lease duration $T^{*}$ increases as shown in Figure 3.d. Now we will explain the effect of $n$ on $U^{*}$. According to (22), $U^{*}=\frac{n}{T^{*}} R^{m}$. As $n$ increases, $U^{*}$ increases. However, with increase in $n, T^{*}$ increases which leads to decrease in $\mathcal{R}^{*}$. Because of these two competing factors, $U^{*}$ first increases and then decreases with increase in $n$. If the number of operators models the amount of competition in a market, this numerical study shows a well known fact: "neither too high nor too low competition is good for overall performance".

\section{B. Heterogeneous Market: Special Case}

We start this section by plotting a typical objective function $U(T)$ for the special case of heterogeneous market. For such a market, $U(T)=\frac{s}{T} \mathcal{R}(s, T)$ (see (20)) where $s$ is a function of $T$. Assuming that $R_{k}^{m}$ 's are sorted in ascending order, $s$ for a given $T$ can be computed as follows:

1) Find $k$ such that $\bar{T}_{k} \leq T<\bar{T}_{k+1}$ where $\bar{T}_{k}=\frac{R_{k}^{m}}{\mu}$.

2) Clearly, operators with index greater than $k$ are not interested. The set of interested operators $\mathcal{S}=$ $\left\{i: i \leq k ; \mathcal{R}(k, T) \geq R_{i}^{m}\right\}$. Hence, $s=|\mathcal{S}|$.

A typical plot of $U(T)$ is shown in Figure 4. The discontinuous and non-smooth nature of $U(T)$ clearly demonstrates that OP1 is not a trivial optimization problem even for the special case of heterogeneous market. Figure 4 contains three arrows showing the intervals where $U(T)=0$. The interval denoted by the green arrow is obvious because the lease duration in this interval is too short to satisfy MERR of any operator even if they were alone in the market. For the intervals denoted by the red arrows, the lease duration is long enough to satisfy the MERR of few of the operators if they were alone in the 

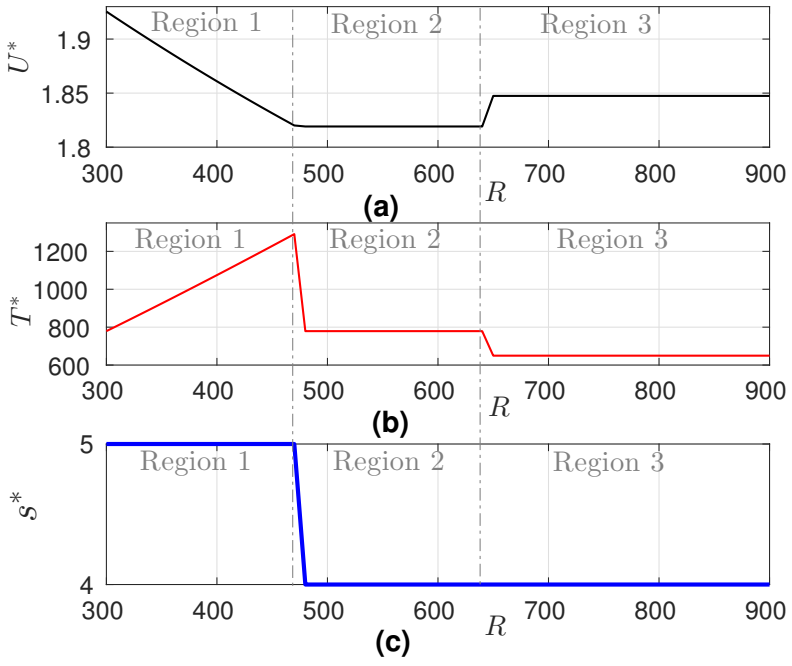

Fig. 5. A numerical study demonstrating the use of lease duration as a control knob that decides which operators enter the market. There are $n=5$ operators. MERR of the first four operators is 300 while that of the fifth operator is $R$. We study the effect of $R$ on the optimal characteristics $U^{*}$, $T^{*}$ and $s^{*}=\left|\mathcal{S}^{*}\right|$ where $\mathcal{S}^{*}$ is the optimal set of interested operators.

market. However, if they were in the market together, MERR of none of the operators are satisfied. As a result, none of the operators are interested. This discussion shows that a large $T$ may not just decrease the objective function but can effectively decrease revenue function of an operator.

Next, we conduct a numerical study to demonstrate the idea of using lease duration as a control knob that decides which operators enter the market. This is shown in Figure 5. There are 5 operators. MERR of the first four operators is 300 while that of the fifth operator is $R$. In Region 1, an increase in $R$ increases $T^{*}$ which in turn decreases $U^{*}$. Though $U^{*}$ decreases, it is still more than the optimal value of the objective function possible with just four operators. Hence, $T^{*}$ is such that all the five operators are interested $\left(s^{*}=5\right)$. As $R$ increases further, the decrease in $U^{*}$ is substantial. Hence, in Regions 2 and 3, MERR of the fifth operator is not satisfied $\left(s^{*}=4\right)$ by decreasing $T^{*} \cdot T^{*}$ in Region 2 is greater than that in Region 3. This can be explained using the same logic as in Section III-B. Let $\widetilde{T}_{4}$ and $\widetilde{T}_{5}$ be the solution to $\mathcal{R}\left(4, \widetilde{T}_{4}\right)=300$ and $\mathcal{R}\left(5, \widetilde{T}_{5}\right)=300$ respectively. $\widetilde{T}_{4}$ $\left(\widetilde{T}_{5}\right)$ is the lease duration to satisfy MERR of the first four operators if the first four operators are interested (all the five operators are interested). In Region $2, \widetilde{T}_{4} \geq \bar{T}$ where $\bar{T}=\frac{R}{\mu}$. Hence, if lease duration is set as $\widetilde{T}_{4}$, MERR of none of the operators will be satisfied making the value of the objective function 0 . Therefore, in Region 2, lease duration is set as $\widetilde{T}_{5}$. In Region $3, \widetilde{T}_{4}<\bar{T}$, and hence lease duration is set as $\widetilde{T}_{4}$. Definitely, $\widetilde{T}_{4} \leq \widetilde{T}_{5}$ (refer to Property 3 and 4 ).

For our next numerical study, we compare the performance of Algorithm 1 with a suboptimal algorithm SUBOP which maximizes the objective function after satisfying MERR of all the $n$ operators. Our setup is as follows. Lets define two clusters/sets $\mathcal{C}_{l}=[100,600]$ and $\mathcal{C}_{h}=[1300,1800] . R_{k}^{m}$ 's are

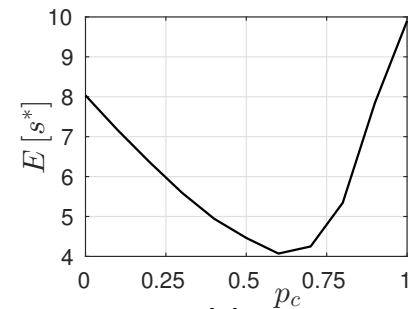

(a)

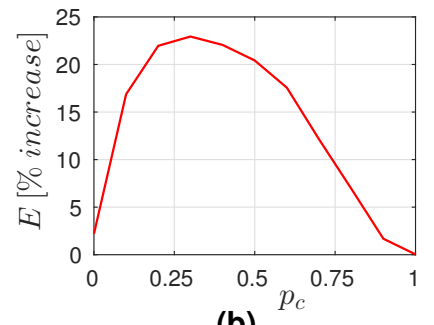

(b)
Fig. 6. A numerical study showing the effect of clustering on the mean optimal characteristics. For each $\left(d_{c}, p_{c}\right)$ pair, the mean optimal characteristics has been averaged over 1000 instances of $R_{k}^{m}$. In (b) \% increase is equal to $\frac{U^{*}-U_{s}^{*}}{U^{*}} \cdot 100$ where $U_{s}^{*}$ is the suboptimal value of the objective function obtained using SUBOP.

chosen independently. $R_{k}^{m}$ lies is $\mathcal{C}_{h}$ and $\mathcal{C}_{l}$ with probability $p_{c}$ and $\left(1-p_{c}\right)$ respectively. Depending on whether $R_{k}^{m}$ lies in $\mathcal{C}_{l}$ or $\mathcal{C}_{h}$, its value is decided at random from the uniform distribution $\mathcal{U}(100,600)$ or $\mathcal{U}(1300,1800)$ respectively. As $p_{c}$ tends to either 0 or $1, R_{k}^{m}$ 's tends to lie mostly in $\mathcal{C}_{l}$ or $\mathcal{C}_{h}$ respectively. When most of the $R_{k}^{m}$ 's lies in one cluster, the scenario is similar to an homogeneous market because the $R_{k}^{m}$ 's are very close to each other. As discussed in Section III-A, in a homogeneous market, the MERR of all the operators needs to be satisfied in order to maximize the objective function. Therefore, the performance of SUBOP and Algorithm 1 are similar as $p_{c}$ tends to either 0 or 1 . Both Figure 6.a and 6.b shows this trend. As $p_{c}$ tends closer to 0 or 1 , the mean optimal number of interested operators tends to $n=10$ and the mean percentage increase of Algorithm 1 compared to SUBOP is almost 0 . However, if $p_{c}$ is not too high, nor too low, the market becomes more heterogeneous. In a heterogeneous market, it may not be optimal to satisfy the MERR of all the operators (as shown in Figure 6.a). Therefore, Algorithm 1 outperforms SUBOP as the market becomes more heterogeneous (as shown in Figure 6.b).

\section{CONCLUSION}

The duration of a spectrum lease is a critical parameter that influences the efficiency of spectrum utilization. In this paper, we study a market scenario where an operator's revenue is a measure of its spectrum utilization. To study the effect of lease duration on spectrum utilization, we present a simple system model which captures interesting properties of spectrum market. Based on the system model, we formulate our optimization problem whose key idea is to use lease duration as a control knob that implicitly decides which wireless operators enter the market. We design optimization algorithms to solve the optimization problem for two special cases. We then use the optimization algorithm to numerically study the variation of optimal characteristics as a function of market parameters. To conclude, we lay the mathematical foundation to optimize the duration of spectrum lease in order to maximize spectrum utilization.

Our immediate future work will be to design optimization algorithms for a general heterogeneous market which is challenging because it may not be possible to reduce the size 
of the set of interested operators like we did in Proposition 2. Generalization of our system model to multiple channel case and capturing transaction costs involved with channel reallocation forms other avenues of our future work. Other extensions to this work involve: (a) Including variance in our system model to capture risk aversion of the operators. (b) Including second price auctions in our system model to capture the variable market-dependent price of a spectrum lease.

\section{APPENDIX A}

PROOF OF PROPOSITION 1

We start with (4) to derive an expression for $\mathcal{R}_{k}(T)$. Consider the term $E\left[\sum_{t=1}^{T} x_{k}(t) \mid I_{k}=1\right]$ of (4). We have,

$$
\begin{aligned}
& E\left[\sum_{t=1}^{T} x_{k}(t) \mid I_{k}=1\right] \\
& =\sum_{\theta} \theta P\left[\sum_{t=1}^{T} x_{k}(t)=\theta \mid I_{k}=1\right] \\
& =\sum_{\theta} \theta \frac{P\left[I_{k}=1 \mid \sum_{t=1}^{T} x_{k}(t)=\theta\right] \cdot P\left[\sum_{t=1}^{T} x_{k}(t)=\theta\right]}{P\left[I_{k}=1\right]}
\end{aligned}
$$

Equation 25 is obtained using Bayes' Theorem. If channel allocation is governed by (3), then the event $I_{k}=1$ is equivalent to $\bigcap_{j \neq k, j \in \mathcal{S}(T)} \sum_{t=1}^{T} x_{k}(t) \geq \sum_{t=1}^{T} x_{j}(t)$ where $\bigcap$ is the AND operator. Hence, the term $P\left[I_{k}=1 \mid \sum_{t=1}^{T} x_{k}(t)=\theta\right]$ of (25) can be written as

$$
\begin{aligned}
& P\left[I_{k}=1 \mid \sum_{t=1}^{T} x_{k}(t)=\theta\right] \\
= & P\left[\bigcap_{j \neq k, j \in \mathcal{S}(T)} \sum_{t=1}^{T} x_{k}(t) \geq \sum_{t=1}^{T} x_{j}(t) \mid \sum_{t=1}^{T} x_{k}(t)=\theta\right] \\
= & P\left[\bigcap_{j \neq k, j \in \mathcal{S}(T)} \sum_{t=1}^{T} x_{j}(t) \leq \theta\right] \\
= & \prod_{j \neq k, j \in \mathcal{S}(T)} P\left[\sum_{t=1}^{T} x_{j}(t) \leq \theta\right]
\end{aligned}
$$

Equation 26 holds because that revenue process of any two operators are not correlated and are hence independent. Using (4), (25) and (26) we get,

$$
\begin{gathered}
\mathcal{R}_{k}(T)=\sum_{\theta} \theta\left(\prod_{j \neq k, j \in \mathcal{S}(T)} P\left[\sum_{t=1}^{T} x_{j}(t) \leq \theta\right]\right. \\
\left.\cdot P\left[\sum_{t=1}^{T} x_{k}(t)=\theta\right]\right)
\end{gathered}
$$

Let $f_{i}(\theta, T)$ and $F_{i}(\theta, T)$ be the probability density function and cummulative distribution function of random variable $\sum_{t=1}^{T} x_{i}(t)$ respectively. Therefore, $P\left[\sum_{t=1}^{T} x_{i}(t)=\theta\right]=$ $f_{i}(\theta, T) d \theta$ and $P\left[\sum_{t=1}^{T} x_{i}(t) \leq \theta\right]=F_{i}(\theta, T)$ which when substituted in (27) yields (9). Now we have to find expressions for $f_{i}(\theta, T)$ and $F_{i}(\theta, T)$. Note that $\sum_{t=1}^{T} x_{i}(t)$ is a Gaussian random variable. This is because $x_{i}(t)$ is a Gaussian and the sum of Gaussian is a Gaussian. Hence, $f_{i}(\theta, T)$ and $F_{i}(\theta, T)$ are completely characterized by the mean $\mu_{i}^{S}(T)$ and variance $\sigma_{i}^{S}(T)$ of $\sum_{t=1}^{T} x_{i}(t)$ as given by (7) and (8) respectively. Finally, we have to find expressions for $\mu_{i}^{S}(T)$ and $\sigma_{i}^{S}(T)$. We have,

$$
\mu_{i}^{S}(T)=E\left[\sum_{t=1}^{T} x_{i}(t)\right]=\sum_{t=1}^{T} E\left[x_{i}(t)\right]=\mu_{i} T
$$

For a first order AR process as governed by (1), $x_{i}(t)$ can be expressed as

$$
x_{i}(t)=a_{i}^{t} x_{i}(0)+\sum_{p=0}^{t-1} a_{i}^{t-1-p} \varepsilon_{i}(p)
$$

Equation 29 can be easily proved using mathematical induction. Also,

$$
\begin{aligned}
\sum_{t=1}^{T} x_{i}(t) & =\sum_{t=1}^{T}\left(a_{i}^{t} x_{i}(0)+\sum_{p=0}^{t-1} a_{i}^{t-1-p} \varepsilon_{i}(p)\right) \\
& =x_{i}(0) \sum_{t=1}^{T} a_{i}^{t}+\sum_{t=1}^{T} \sum_{p=0}^{t-1} a_{i}^{t-1-p} \varepsilon_{i}(p) \\
& =x_{i}(0) \sum_{t=1}^{T} a_{i}^{t}+\sum_{p=0}^{T-1} \sum_{t=p+1}^{T} a_{i}^{t-1-p} \varepsilon_{i}(p) \\
& =x_{i}(0) \frac{a_{i}-a_{i}^{T+1}}{1-a_{i}}+\sum_{p=0}^{T-1} \frac{1-a_{i}^{T-p}}{1-a_{i}} \varepsilon_{i}(p)
\end{aligned}
$$

Equation 30 is obtained using (29). Equation 31 is obtained by changing the order of summation. Now, $\sigma_{i}^{S}(T)=$

$$
\begin{aligned}
\sqrt{\operatorname{Var}\left[\sum_{t=1}^{T} x_{i}(t)\right]} \text { where, } \\
\operatorname{Var}\left[\sum_{t=1}^{T} x_{i}(t)\right] \\
=\operatorname{Var}\left[x_{i}(0) \frac{a_{i}-a_{i}^{T+1}}{1-a_{i}}+\sum_{p=0}^{T-1} \frac{1-a_{i}^{T-p}}{1-a_{i}} \varepsilon_{i}(p)\right] \\
=\left(\frac{a_{i}-a_{i}^{T+1}}{1-a_{i}}\right)^{2} \operatorname{Var}\left[x_{i}(0)\right] \\
+\sum_{p=0}^{T-1}\left(\frac{1-a_{i}^{T-p}}{1-a_{i}}\right)^{2} \operatorname{Var}\left[\varepsilon_{i}(p)\right]
\end{aligned}
$$




$$
\begin{aligned}
& =\left(\frac{a_{i}-a_{i}^{T+1}}{1-a_{i}}\right)^{2} \sigma_{i}^{2}+\sum_{p=0}^{T-1}\left(\frac{1-a_{i}^{T-p}}{1-a_{i}}\right)^{2}\left(\sigma_{i}^{\varepsilon}\right)^{2} \\
& =\frac{T-a_{i}\left(2-2 a_{i}^{T}+a_{i} T\right)}{\left(1-a_{i}\right)^{2}} \cdot \frac{\left(\sigma_{i}^{\varepsilon}\right)^{2}}{1-a_{i}^{2}} \\
& =\frac{T-a_{i}\left(2-2 a_{i}^{T}+a_{i} T\right)}{\left(1-a_{i}\right)^{2}} \sigma_{i}^{2}
\end{aligned}
$$

Equation 33 is obtained from (32). Equation 34 holds because $\varepsilon_{i}(p)$ are independent random variables. Equations 35 and 36 follows from the definition of $\sigma_{i}^{\varepsilon}$ and $\sigma_{i}$ as given by (2) and the paragraph before it. Finally, (28) and (36) are same as (5) and (6) respectively. This completes the proof.

\section{REFERENCES}

[1] V. Cisco Mobile, "Cisco visual networking index: Global mobile data traffic forecast update, 2016-2021 white paper," 2017.

[2] I. F. Akyildiz, W.-Y. Lee, M. C. Vuran, and S. Mohanty, "Next generation/dynamic spectrum access/cognitive radio wireless networks: A survey," Computer networks, vol. 50, no. 13, pp. 2127-2159, 2006.

[3] M. A. McHenry, P. A. Tenhula, D. McCloskey, D. A. Roberson, and C. S. Hood, "Chicago spectrum occupancy measurements \& analysis and a long-term studies proposal," in Proceedings of the first international workshop on Technology and policy for accessing spectrum. ACM, 2006, p. 1.

[4] M. R. Hassan, G. C. Karmakar, J. Kamruzzaman, and B. Srinivasan, "Exclusive use spectrum access trading models in cognitive radio networks: A survey," IEEE Communications Surveys \& Tutorials, vol. 19, no. 4, pp. 2192-2231, 2017.

[5] P. Milgrom, E. G. Weyl, and A. L. Zhang, "Redesigning spectrum licenses," Regulation, vol. 40, p. 22, 2017.

[6] Federal Communications Commission, "Amendment of the commission's rules with regard to commercial operations in the 3550-3650 MHz band," May 2016. [Online]. Available: https://apps.fcc.gov/edocs_public/attachmatch/FCC-16-55A1.pdf

[7] Q. Zhao and B. M. Sadler, "A survey of dynamic spectrum access," Signal Processing Magazine, IEEE, vol. 24, no. 3, pp. 79-89, 2007.

[8] E. Ahmed, A. Gani, S. Abolfazli, L. J. Yao, and S. U. Khan, "Channel assignment algorithms in cognitive radio networks: Taxonomy, open issues, and challenges," IEEE Communications Surveys \& Tutorials, vol. 18, no. 1, pp. 795-823, 2014.

[9] T. Wysocki and A. Jamalipour, "Sharpe ratio based pricing of cognitive radio access," in Wireless Communications and Networking Conference (WCNC), 2011 IEEE. IEEE, 2011, pp. 2119-2124.

[10] Y. Zhang, C. Lee, D. Niyato, and P. Wang, "Auction approaches for resource allocation in wireless systems: A survey," IEEE Communications surveys \& tutorials, vol. 15, no. 3, pp. 1020-1041, 2013.

[11] M. Saint and T. X. Brown, "The dynamic policy license," in Dynamic Spectrum Access Networks (DySPAN), 2017 IEEE International Symposium on. IEEE, 2017, pp. 1-9.

[12] A. Nika, Z. Zhang, B. Y. Zhao, and H. Zheng, "Toward practical spectrum permits," IEEE Transactions on Cognitive Communications and Networking, vol. 3, no. 1, pp. 112-122, 2017.

[13] Y. Saito, K. Yamamoto, H. Murata, and S. Yoshida, "Joint dynamics of spectrum allocation and user behavior in spectrum markets," in Global Telecommunications Conference, 2009. GLOBECOM 2009. IEEE. IEEE, 2009, pp. 1-5.

[14] M. R. Hassan, G. Karmakar, and J. Kamruzzaman, "Reputation and user requirement based price modeling for dynamic spectrum access," Mobile Computing, IEEE Transactions on, vol. 13, no. 9, pp. 2128-2140, 2014.
[15] O. Simeone, I. Stanojev, S. Savazzi, Y. Bar-Ness, U. Spagnolini, and R. Pickholtz, "Spectrum leasing to cooperating secondary ad hoc networks," IEEE Journal on Selected Areas in Communications, vol. 26, no. $1,2008$.

[16] S. D. Jones, E. Jung, X. Liu, N. Merheb, and I.-J. Wang, "Characterization of spectrum activities in the us public safety band for opportunistic spectrum access," in New Frontiers in Dynamic Spectrum Access Networks, 2007. DySPAN 2007. 2nd IEEE International Symposium on. IEEE, 2007, pp. 137-146.

[17] Y.-C. Liang, Y. Zeng, E. C. Peh, and A. T. Hoang, "Sensing-throughput tradeoff for cognitive radio networks," Wireless Communications, IEEE Transactions on, vol. 7, no. 4, pp. 1326-1337, 2008.

[18] A. MacKay, "The structure of costs and the duration of supplier relationships," Citeseer, Tech. Rep., 2016.

[19] C. J. Ellis and S. Holden, "Optimal contract length in a reputational model of monetary policy," European Economic Review, vol. 41, no. 2, pp. 227-243, 1997.

[20] R. Tse, "Lag vacancy, effective rents and optimal lease term," Journal of property investment \& finance, vol. 17, no. 1, pp. 75-88, 1999.

[21] H.-P. Chao and R. Wilson, "Optimal contract period for priority service," Operations research, vol. 38, no. 4, pp. 598-606, 1990.

[22] L. Deek, X. Zhou, K. Almeroth, and H. Zheng, "To preempt or not: Tackling bid and time-based cheating in online spectrum auctions," in INFOCOM, 2011 Proceedings IEEE. IEEE, 2011, pp. 2219-2227.

[23] K. W. Choi and E. Hossain, "Opportunistic access to spectrum holes between packet bursts: A learning-based approach," IEEE Transactions on Wireless Communications, vol. 10, no. 8, pp. 2497-2509, 2011.

[24] J. Bertrand, "Review of walrass théorie mathématique de la richesse sociale and cournots recherches sur les principes mathematiques de la theorie des richesses in cournot oligopoly: Characterization and applications. edited by af daughety," Cournot oligopoly: Characterization and applications, pp. 73-81, 1988.

[25] A.-A. Cournot, Recherches sur les principes mathématiques de la théorie des richesses par Augustin Cournot. chez L. Hachette, 1838.

[26] C. Liu and R. A. Berry, "Competition with licensed shared spectrum." in CISS, 2014, pp. 1-6.

[27] S.-P. Sheng, Y. Liu, and M. Liu, "A regulated oligopoly multi-market model for trading smart data," in Computer Communications Workshops (INFOCOM WKSHPS), 2015 IEEE Conference on. IEEE, 2015, pp. $510-515$.

[28] Y. Chen and H.-S. Oh, "A survey of measurement-based spectrum occupancy modeling for cognitive radios," IEEE Communications Surveys \& Tutorials, vol. 18 , no. 1, pp. 848-859, 2016.

[29] H. T. Luong, "Measure of bullwhip effect in supply chains with autoregressive demand process," European Journal of Operational Research, vol. 180, no. 3, pp. 1086-1097, 2007.

[30] C. Chatfield, The analysis of time series: an introduction. CRC press, 2016.

[31] G. H. Badawy, A. A. Sayegh, and T. D. Todd, "Energy provisioning in solar-powered wireless mesh networks," IEEE Transactions on Vehicular Technology, vol. 59, no. 8, pp. 3859-3871, 2010.

[32] R. Combes and A. Proutiere, "Dynamic rate and channel selection in cognitive radio systems," IEEE Journal on Selected Areas in Communications, vol. 33, no. 5, pp. 910-921, 2015.

[33] Y. Chen, L. Duan, J. Huang, and Q. Zhang, "Balancing income and user utility in spectrum allocation," IEEE Transactions on Mobile Computing, vol. 14, no. 12, pp. 2460-2473, 2015.

[34] J. Bae, E. Beigman, R. A. Berry, M. L. Honig, and R. Vohra, "Sequential bandwidth and power auctions for distributed spectrum sharing," IEEE Journal on Selected Areas in Communications, vol. 26, no. 7, 2008.

[35] G. Saha, A. A. Abouzeid, and M. Matinmikko, "Optimal duration of spectrum lease: A mathematical approach." [Online]. Available: https://www.ecse.rpi.edu/homepages/abouzeid/preprints/lease_duration.pdf 